Note

\title{
Growth Characteristics of Four Fungal Species in Bathrooms
}

\author{
NOBUO HAMADA, AND NIICHIRO ABE \\ Osaka City Institute of Public Health and Environmental Sciences, \\ 8-34 Tojo-cho, Tennoji, Osaka 543-0026, Japan
}

Received 11 March, 2010/Accepted 21 June, 2010

\begin{abstract}
The growth of four fungal species (four isolates) from bathrooms was examined under various environmental conditions. These fungi were found several times in bathrooms and washing machines, but not in other indoor environments such as house dust or windows. The four species (Ramichloridium strelitziae, Cyphellophora laciniata, Phoma fimeti, and Exophiala sp.) were identified using DNA and morphological analyses. These bathroom fungi were able to consume surfactants, soap and shampoo, but were unable to grow well in high-temperature or dry conditions. Soap and shampoo seem to affect fungal flora in the bathroom.
\end{abstract}

Key words : Bathrooms / Detergent/Fungal contamination.

Several genera of fungi with generally slow growth - Exophiala, Phoma, Scolecobasidium, Phialophora, and Cladophialophora - are more common in bathrooms than in other indoor areas such as air-conditioners or on windows, which are also humid and warm environments as bathrooms are (Hamada and Abe, 2008). Growth characteristics of common fungi in bathrooms were described in an earlier paper (Hamada and Abe, 2009) (see Table 1). The six predominant species - Cladophialophora boppii, Exophiala spinifera, E. salmonis, Phialophora europaea, Phoma herbarum, and Scolecobasidium constrictum-grew on media of 0.05 , and $0.25 \%$ nonion surfactant, a component of shampoo, although common airborne indoor fungi such as Aureobasidium sp. and Cladosporium cladosporioides grew on media containing sodium fatty acids. These characteristic fungi found in bathrooms were able to use detergents as a nutrient. On the other hand, these fungi were unable to grow well in $35^{\circ} \mathrm{C}$ or on media with $15 \% \mathrm{NaCl}$, namely under high-temperature or dry conditions. Therefore, factors controlling fungi found commonly in bathrooms are apparently more specifically associated with nutrients than with environments.

${ }^{*}$ Corresponding author. Tel : +81-6-6771-3108, Fax: +81 6-6772-0676, E-mail: n-hamada (a)city.osaka.lg.jp
Fungi found more frequently in bathrooms than in other areas were not only those six species. Other fungi that we had never found in other parts of the household were also found on several occasions in bathrooms and washing machines, where detergents, soaps, and shampoo are usually used.

In this study, four species of fungi isolated from bathroom walls or floors were identified as in an earlier study (Hamada and Abe, 2008). Moreover, their respective growth characteristics in culture on various media and under various environmental conditions were examined to confirm the factors determining fungal species growth in bathrooms.

Identification of four species of fungi according to their genetic and morphological characteristics was attempted in this study.

Genomic DNA from each mycelium sample was extracted and purified using the DNeasy Blood and Tissue Kit according to the manufacturer's instructions, as reported previously (Hamada and Abe, 2009). Subsequently, PCR was performed using the ITS1 (or V9D) and ITS4 primers, which amplify the region of the internal transcribed spacers 1 (ITS1) and 2 (ITS2) including the $5.8 \mathrm{~S}$ ribosomal RNA gene (5.8SrDNA). The PCR products were gel-purified using a QIAquick Gel Extraction Kit and were sequenced in both directions using an $\mathrm{ABI} 3130$ automated sequencer. Sequence chromatograms 
TABLE 1. Effects of three surfactants on the growth of common fungi in dwellings and bathrooms

\begin{tabular}{|c|c|c|c|c|c|c|c|c|c|c|}
\hline \multirow[t]{3}{*}{ Fungal species } & & \multirow{3}{*}{$\begin{array}{c}\text { (Standard) } \\
25^{\circ} \mathrm{C}\end{array}$} & \multirow{2}{*}{\multicolumn{2}{|c|}{ Temperature* }} & \multirow{2}{*}{\multicolumn{2}{|c|}{$\mathrm{NaCl}^{*}$}} & \multicolumn{4}{|c|}{ Surfactant } \\
\hline & & & & & & & \multicolumn{2}{|c|}{ SO (sodium fatty acid) ${ }^{* *}$} & \multicolumn{2}{|c|}{ AE (non-ion surfactant)** } \\
\hline & & & $33^{\circ} \mathrm{C}$ & $35^{\circ} \mathrm{C}$ & $10 \%$ & $15 \%$ & $0.05 \%$ & $0.25 \%$ & $0.05 \%$ & $0.25 \%$ \\
\hline \multicolumn{11}{|c|}{ Aureobasidium sp. ${ }^{a}$} \\
\hline & Count & 40 & 28 & 13 & 34 & 0 & 34 & 13 & 0 & 0 \\
\hline \multirow{2}{*}{\multicolumn{11}{|c|}{ Cladosporium cladosporioides ${ }^{a}$}} \\
\hline & & & & & & & & & & \\
\hline & Count & 40 & 0 & 0 & 40 & 22 & 46 & 8 & 0 & 0 \\
\hline & Size & + & & & + & \pm & + & \pm & & \\
\hline \multicolumn{11}{|c|}{ Cladophialophora boppii ${ }^{-1}$} \\
\hline & Count & 40 & 0 & 0 & 0 & 0 & 34 & 0 & 25 & 26 \\
\hline & Size & + & & & & & + & & + & + \\
\hline \multicolumn{11}{|c|}{ Exophiala spinifera $^{b}$} \\
\hline & Count & 40 & 6 & 0 & 0 & 0 & 39 & 30 & 26 & 21 \\
\hline & Size & + & - & & & & + & \pm & + & + \\
\hline \multicolumn{11}{|c|}{ Exophiala salmonis ${ }^{b}$} \\
\hline & Count & 40 & 0 & 0 & 0 & 0 & 43 & 37 & 46 & 24 \\
\hline & Size & + & & & & & + & \pm & + & + \\
\hline \multicolumn{11}{|c|}{ Phialophora europaea ${ }^{b}$} \\
\hline & Count & 40 & 38 & 0 & 0 & 0 & 34 & 39 & 37 & 29 \\
\hline & Size & + & \pm & & - & & + & \pm & \pm & \pm \\
\hline \multicolumn{11}{|c|}{ Phoma herbarum ${ }^{b}$} \\
\hline & Count & 40 & 41 & 0 & 21 & 0 & 37 & 26 & 37 & 24 \\
\hline & Size & + & \pm & & \pm & & + & + & \pm & - \\
\hline \multicolumn{11}{|c|}{ Scolecobasidium constrictum ${ }^{b}$} \\
\hline & Count & 40 & 0 & 0 & 0 & 0 & 35 & 29 & 32 & 28 \\
\hline & Size & + & & & & & + & + & + & + \\
\hline
\end{tabular}

The growth of each fungus is indicated using the count and size of colonies.

$a, b$ in fungal species indicated common fungi in dwellings or in bathrooms.

The count of each fungal colony under various culture conditions was compared with that on 1/4PDA at $25^{\circ} \mathrm{C}$ indicated as 40 .

${ }^{*}$ Colony size was compared with that on $1 / 4 \mathrm{PDA}$ at $25^{\circ} \mathrm{C}$, and assessed as follows: + , same; \pm , smaller; - , much smaller

${ }^{* *}$ Colony size was compared with that on $0.05 \%$ SO, and assessed as follows: + , same; \pm , smaller; - , much smaller

This table summarizes the results of an earlier study (Hamada and Abe, 2009).

from each strand were inspected using software (Sequencher ver. 4.1). Homology search using the obtained partial gene sequences was performed with FASTA at DDBJ (http://www.ddbj.nig.ac.jp/search/ fasta-j.html).

The nucleotide sequence data reported in this paper are available in the International Nucleotide Sequence Database (INSD, GenBank/EMBL/DDBJ) under accession numbers AB488487-AB488490; those voucher cultures were also deposited in the JCM.

The following four species of fungi were collected from bathroom walls or floors (Hamada and Abe, 2008), identified and subjected to examination of growth characteristics: Ramichloridium strelitziae (NH102, AB488487, JCM16191), Cyphellophora laciniata (NH404, AB488488, JCM16192), Phoma fimeti (NH650, AB488489, JCM16193), and
Exophiala sp. (NH1238, AB488490, JCM16194).

After blocks of collected spores were diluted to 1,000 or 10,000 times, suspensions of spores of the four species were inoculated onto media for cultivation. About $25 \mathrm{ml}$ of $1 / 4$ potato-dextrose agar (PDA) was added onto a Petri dish and used as corresponding medium (Hamada and Abe, 2008).

Each fungus, growing slowly, was cultured for 8-14 days in an incubator maintained variously at a temperature of 25 (standard), $30^{\circ} \mathrm{C}, 33^{\circ} \mathrm{C}$ or $35^{\circ} \mathrm{C}$. The temperature deviation was within $1.0^{\circ} \mathrm{C}$ (Hamada and Abe, 2009). A culture of $1 / 4$ PDA with 5,10 , or $15 \%$ $\mathrm{NaCl}$ added was used to examine the tolerance of fungi to dry conditions. For comparison of the effect of $\mathrm{NaCl}$, each fungus was incubated in darkness for 8-14 days at $25^{\circ} \mathrm{C}$. A culture of $1 / 4$ PDA was also used as the standard of acidic medium to examine the tolerance of fungi to alkali conditions. To adjust 


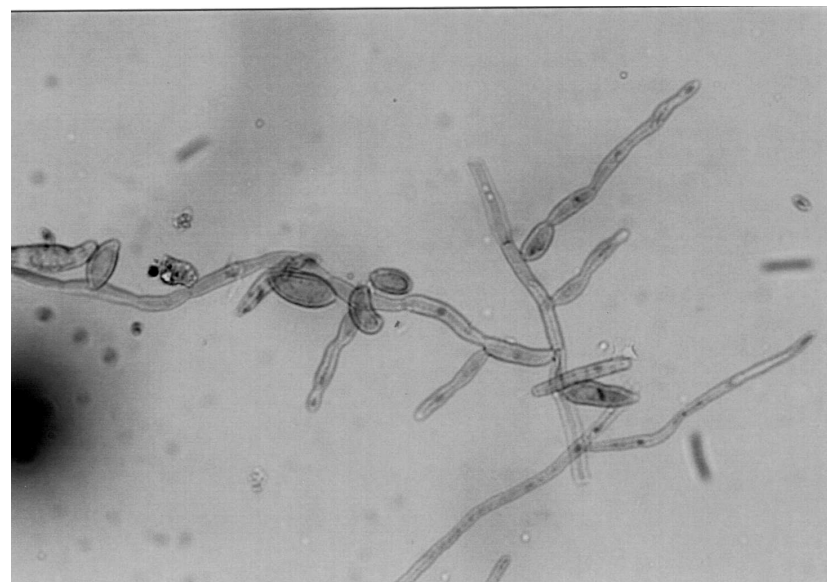

FIG. 1. Exophiala sp. (JCM16194) $(\times 400)$

Spores: oval, often falcate.

the $\mathrm{pH}$ value of the medium to an alkali level, a modified version of the method of Nagai et al. (1998) was used. The $\mathrm{pH}$ of $1 / 4$ PDA was 6.0 (same with $25^{\circ} \mathrm{C}$ ); that of the alkaline 1/4 PDA was 9.7.

Moreover, media containing three main components of commercial detergents were used for cultivation. These components were used as culture media: SO (sodium oleate, sodium fatty acid); $A E$ (polyoxyethylene (9) lauryl ether, nonion surfactant); and LAS (sodium dodecyl benzenesulfonate, anion surfactant), as used in an earlier study (Hamada and
Abe, 2009). These three surfactants were each adjusted to $0.01 \%, 0.05 \%$, and $0.25 \%(0.10 \mathrm{~g}, 0.50 \mathrm{~g}$, $2.5 \mathrm{~g} / \mathrm{L}$ ) and added to $15 \mathrm{~g} / \mathrm{L}$ of Bacto agar. On various surfactant components, growth characteristics of those four fungi were compared in terms of counts, colony sizes, and colors. The fungi inoculated onto Petri dishes with various added surfactant components were incubated at $25^{\circ} \mathrm{C}$ for $12-16$ days.

To count the colonies found on each medium, media with 10-100 colonies were selected from the cultures that had been inoculated at various levels of dilution. The fungal count of each species shown in the tables and detected on $1 / 4$ PDA at $25^{\circ} \mathrm{C}$ under the corresponding conditions was around 40 colonies.

The predicted size fragment (approximately 500$700 \mathrm{bp}$, depending upon the isolate analyzed) was amplified using PCR in black yeast isolates (data not shown). Homology search using the obtained partial sequences revealed that the sequences of isolates $\mathrm{NH} 102$ (within 533 bp of overlap), NH 404 (within 597 bp of overlap), NH650 (within 539 bp of overlap), and $\mathrm{NH} 1238$ (within $601 \mathrm{bp}$ of overlap) had similarity of $93.2 \%, 98.8 \%, 96.1 \%$, and $98.8 \%$, respectively, with Ramichloridium strelitziae (EU041803), Cyphellophora laciniata (EU035416), Phoma fimeti (AY531687), and Exophiala sp. (FJ665274).

TABLE 2. Effects of environmental factors on the growth of four fungi

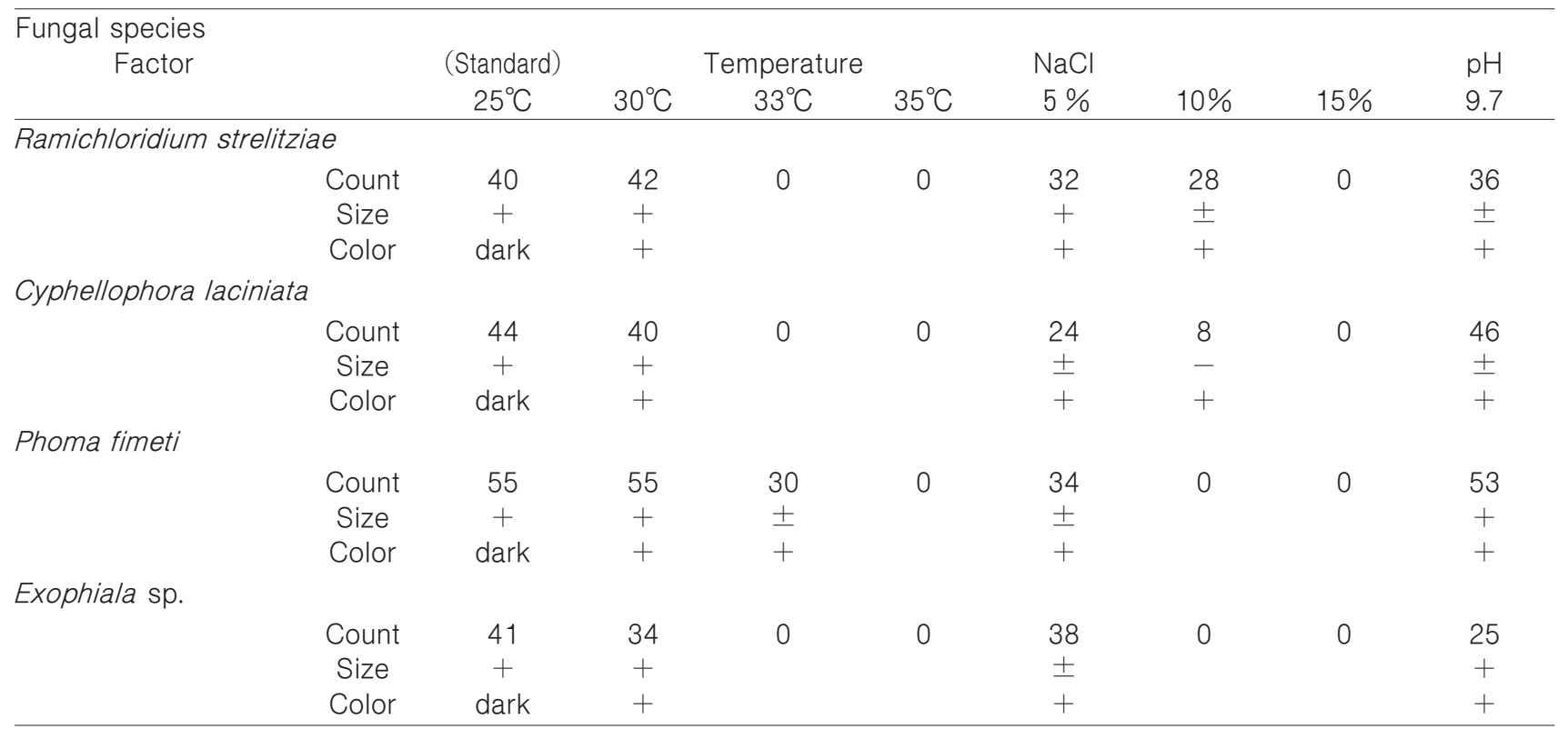

Fungal count indicates the total number of colonies detected on each of two Petri dishes.

Size of colonies was compared with that on $1 / 4 \mathrm{PDA}$ at $25^{\circ} \mathrm{C}, \mathrm{pH} 6.0$, and assessed as follows: ++ , larger; + , same; \pm , smaller; -, much smaller

Color of fungi was compared with that on $1 / 4 \mathrm{PDA}$ at $25^{\circ} \mathrm{C}, \mathrm{pH} 6.0$, and assessed as follows: ++ , darker; + , same; \pm , lighter 
A direct preparation of dark mold isolated and cultured on a slant appeared dark under microscopic observation. This fungus was identified morphologically using a fungi monograph (de Hoog et al., 2000; Arzanlou et al., 2007; Boerema et al., 2004), Ramichloridium strelitziae were colonies with red pigments, with unbranched erect brown conidiophores, and subhyaline aseptate clavate conidia. Cyphellophora laciniata were dark colonies, with intercalary phialides, sickle-shaped conidia, and dark 1-3 septate. Phoma fimeti were slowly growing colonies with gray aerial mycelia, pycnidia subglobose with 60-160 $\mu \mathrm{m}$ diameter and ellipsoidal conidia. Exophiala sp. (see Fig. 1) were dark velvety colonies, with cylindrical conidiogenous cells, and oval to falcate conidia. Exophiala sp. was identified as E. equina by Dr. de Hoog, CBS (personal communication, soon to be published).

The colony counts of four species cultured at 30 ${ }^{\circ} \mathrm{C}$ were similar to those at $25^{\circ} \mathrm{C}$ (Table 2). The sizes and color of all species that were examined was similar at $25^{\circ} \mathrm{C}$ and $30^{\circ} \mathrm{C}$. At $33^{\circ} \mathrm{C}$, only colonies of $P$. fimeti were detected, although the counts and sizes of colonies were smaller. Colonies of four species were detected on media with $5 \% \mathrm{NaCl}$, but their colony count was smaller than on media without $\mathrm{NaCl}$. Moreover, only colonies of $R$. strelitziae and $C$. laciniata were detected on media with $10 \% \mathrm{NaCl}$; those counts and sizes decreased with the addition of $\mathrm{NaCl}$. In the three fungi $-R$. strelitziae, C. laciniata and $P$. fimet $i$ - the colony counts were approximately similar on media of pH 6.0 and $\mathrm{pH}$ 9.7. The sizes of $P$. fimeti and Exophiala sp. were similar on media of both $\mathrm{pH}$, although the colors of all four species were similar on those media.

The effects of the sodium fatty acid SO on the growth of the four fungi were examined (Table 3 ). Colonies of all examined species were detected on media of $0.01 \%, 0.05 \%$, and $0.25 \%$ SO within 16 days. The colony count on media of $0.25 \%$ SO compared to that on 1/4 PDA was lower in four species, and less than one-third of that on 1/4 PDA for $C$. laciniata and $P$ fimeti. Moreover, the colonies of $C$. laciniata were much smaller than on $0.05 \%$ SO. However, the color of colonies of $R$. strelitziae and $P$. fimeti darkened concomitantly with increased $S O$ concentration.

No C. laciniata colony was detected on $0.25 \% \mathrm{AE}$, but colonies of $R$. strelitziae, $P$. fimeti, and Exophiala sp. on $0.25 \%$ AE were detected, although the colony count in P. fimeti was also lower.

The color of the colonies of $R$. strelitziae was darker on $0.05 \% \mathrm{AE}$ than on $0.01 \% \mathrm{AE}$, and colonies on $0.25 \% \mathrm{AE}$ were darker than those on $0.05 \% \mathrm{AE}$ (Table 3), different from $P$. fimeti with a color on $0.25 \% \mathrm{AE}$ that was lighter than on $0.05 \% \mathrm{AE}$ (Fig. 2).

TABLE 3. Effects of three surfactants on the growth of four fungi

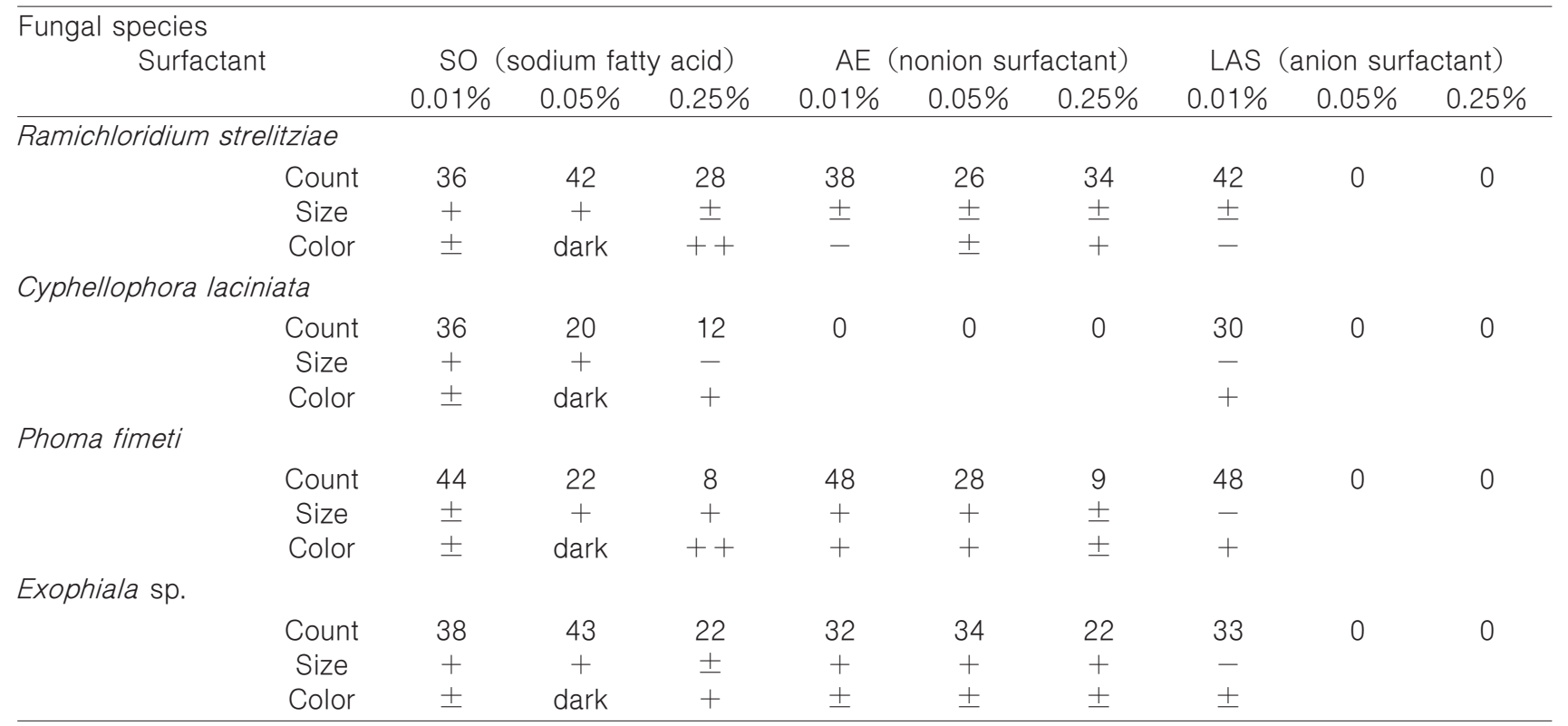

Fungal count shows the total number of colonies detected on each of two Petri dishes, on which the suspension of spores used in Table 2 was inoculated.

Colony size was compared with that on $0.05 \%$ SO, and assessed as follows: ++ , larger; + , same; \pm , smaller; - , much smaller

Color of fungi was compared with that on $0.05 \%$ SO and assessed as follows: ++ , darker; + , same; \pm , lighter 


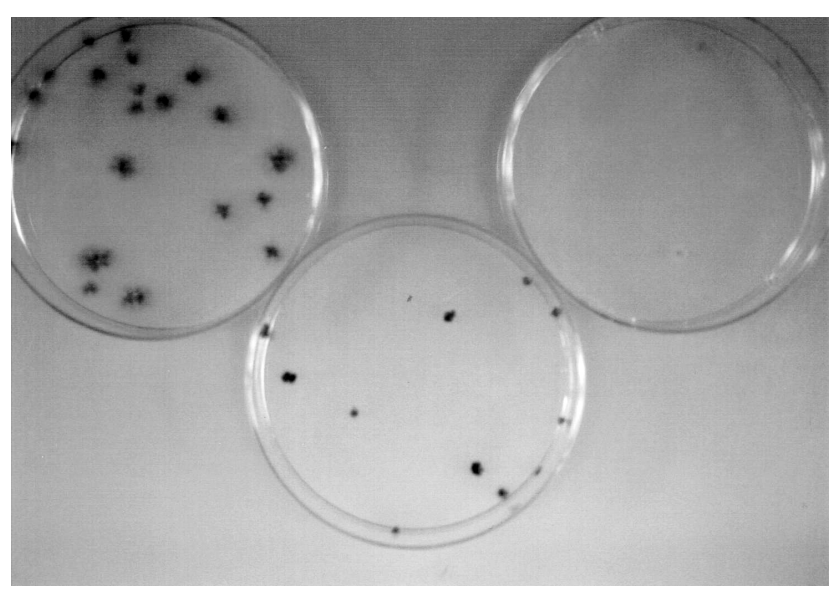

FIG. 2. Growth of Phoma fimeti (JCM16193) cultured on $\mathrm{AE}$ (nonion surfactant) medium for 14 days.

Left, 0.01\% AE; center, 0.05\% AE; right, 0.25\% AE

The colony counts of fungi were $30-48$ on $0.01 \%$ LAS and were similar to those recorded on the other surfactant media, $0.01 \% \mathrm{SO}$ and $0.01 \% \mathrm{AE}$. The colony, however was smaller than on the other media, irrespective of the fungal species that was examined.

All four species examined in this study were grown on media of $0.25 \% \mathrm{SO}$ and with $\mathrm{pH} 9.7$ (Table 2). Therefore, all four fungi that were examined are apparently alkali-tolerant. Those fungi are thought to use soap as a nutrient. Soap apparently has a strong effect on fungal contamination in bathrooms.

The three species, $R$. strelitziae, P. fimeti, and Exophiala sp., grew on $0.25 \%$ AE media (Table 3), as did six species using detergent described in an earlier report (Hamada and Abe, 2008). The fungi that grow on $0.25 \%$ AE or $0.25 \%$ SO media would seem therefore to make use of the shampoo or soap adhering to or condensed on the walls and floors. Shampoo containing nonion surfactant is therefore thought to have a clear effect on the fungal flora in bathrooms. Some fungi in bathrooms use shampoo, a synthetic surfactant, as a nutrient, which is interesting from an evolutional viewpoint.

However, the growth rate of $R$. strelitziae and $P$. fimeti on $0.25 \%$ AE was lower than on $0.05 \% \mathrm{SO}$, different from most bathroom fungi described before (Table 1). Consequently, these fungi were thought to be not as common in bathrooms as the previous six species. It is notable that $C$. laciniata cannot use nonion surfactant, different from the other three fungi. Moreover, C. laciniata grows less well than the other three fungi on soap medium: the colonies were much smaller on $0.25 \%$ SO. In fact, C. laciniata was often found in both bathrooms and washing machines, although C. laciniata was misidentified as Fusariella sp. in some earlier studies (Hamada and Abe, 2008 ; Hamada, 2005). Why C. laciniata, which grows slowly, is often found in bathrooms and washing machines remains unclear.

However, fungi were unable to grow on anion medium, including more than $0.01 \%$ LAS, which is similar to the 13 most common species collected and examined (Hamada and Abe, 2009).

The four species examined in this study did not grow at $35^{\circ} \mathrm{C}$ (high temperature) or on media with $15 \% \mathrm{NaCl}$ (osmotic conditions), as did the six species of bathroom fungi investigated in the previous study (Table 1). These fungi seemed to prefer water at temperatures lower than $30^{\circ} \mathrm{C}$, and did not flourish with hot water used in bathtubs or in house dust in dwellings as well as changeable outdoor environments. Consequently, these four fungi, growing slowly, might have difficulty surviving in environments other than most places associated with detergent use such as those of bathrooms, washing machines, and sinks of kitchens.

This study of characteristics of common bathroom fungi suggests a means of controlling fungal contamination in bathrooms. Washing away soap and shampoo, which provide nutrients for fungal contamination, is most important. Drying treatments in bathrooms are also thought to be effective for eliminating contamination.

\section{REFERENCES}

Arzanlou, M., Groenewald, J.Z., Gams, W., Braun, U., Shin, H.D., and Crous, P.W. (2007), Phylogenetic and morphotaxonomic revision of Ramichloridium and allied genera. Studies in Mycology, 58, 57-93.

Boerema, G..H., de Gruyter, J., Noordeloos, M.E., and Hamers, M.E.C. (2004), Phoma identification manual. CABI Publishing, Cambridge.

de Hoog, G..S., Guarro, J., Gene, J., and Figueras, M.J. (2000), Atlas of clinical fungi, 2nd ed., Centraalbureau voor Schimmelcultures, Utrecht.

Hamada, N. (2005), Growth on various detergent components of fungi found in washing machines. Seikatsu Eisei, 49, 161-167. (in Japanese)

Hamada, N., and Abe, N. (2008), Characteristics of recent fungal contamination in bathrooms: examination of fungal and yeasty flora. Seikatsu Eisei, 52, 98-106. (in Japanese)

Hamada, N., and Abe, N. (2009), Physiological characteristics of 13 common fungal species in bathrooms. Mycoscience, 50, 421-429.

Nagai, K., Suzuki, K., and Okada, G. (1998) Studies on the distribution of alkalophilic and alkali-tolerant soil fungi II: fungal flora in two limestone caves in Japan. Mycoscience, 39, 293-298. 